CRYSTALLOGRAPHIC COMMUNICATIONS

ISSN 2056-9890

\section{Hydrogen bonds and van der Waals forces as tools for the construction of a herringbone pattern in the crystal structure of hexane-1,6-diaminium hexane- 1,6-diyl bis(hydrogen phosphonate)}

\author{
Guido J. Reiss,* Martin van Megen and Walter Frank
}

Received 27 November 2016

Accepted 13 December 2016

Edited by M. Weil, Vienna University of
Technology, Austria

Keywords: crystal structure; hydrogen bonding; phosphonates; herringbone pattern; crystal engineering.

CCDC reference: 1522538

Supporting information: this article has supporting information at journals.iucr.org/e

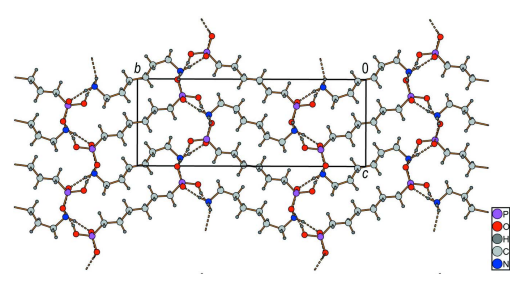

OPEN $\odot$ ACCESS
Institut für Anorganische Chemie und Strukturchemie, Lehrstuhl II: Material- und Strukturforschung, Heinrich-HeineUniversität Düsseldorf, Universitätsstrasse 1, D-40225 Düsseldorf, Germany. *Correspondence e-mail: reissg@hhu.de

The asymmetric unit of the title salt, $\left[\mathrm{H}_{3} \mathrm{~N}\left(\mathrm{CH}_{2}\right)_{6} \mathrm{NH}_{3}\right]\left[(\mathrm{HO}) \mathrm{O}_{2} \mathrm{P}\left(\mathrm{CH}_{2}\right)_{6}\right.$ $\left.\mathrm{PO}_{2}(\mathrm{OH})\right]$, consists of one half of a hexane-1,6-diaminium dication and one half of a hexane-1,6-diyl bis(hydrogen phosphonate) dianion. Both are located around different centres of inversion (Wyckoff sites: $2 a$ and $2 d$ ) of the space group $P 2_{1} / c$. The shape of the hexane-1,6-diaminium cation is best described as a double hook. Both aminium groups as well as the two attached $\mathrm{CH}_{2}$ groups are turned out from the plane of the central four $\mathrm{C}$ atoms. In contrast, all six $\mathrm{C}$ atoms of the dianion are almost in a plane. The hydrogen phosphonate $\left(-\mathrm{PO}_{3} \mathrm{H}\right)$ groups of the anions and the aminium groups of the cations form twodimensional $\mathrm{O}-\mathrm{H} \cdots$ and $\mathrm{O}-\mathrm{H} \cdots \mathrm{N}$ hydrogen-bonded networks parallel to the $a c$ plane, built up from ten-membered and twelve-membered ring motifs with graph-set descriptors $R_{3}^{3}(10)$ and $R_{5}^{4}(12)$, respectively. These networks are linked by the alkylene chains of the anions and cations. The resulting three-dimensional network shows a herringbone pattern, which resembles the parent structures 1,6-diaminohexane and hexane-1,6-diphosphonic acid.

\section{Chemical context}

Salts which comprise organophosphonate anions and organic cations, e.g. protonated primary (Mahmoudkhani \& Langer, $2002 a, b, c)$, secondary (Wheatley et al., 2001) or tertiary amines (Kan \& Ma, 2011) are of growing interest in supramolecular chemistry and crystal engineering. Compounds of this type possess interesting topologies and an extended structural diversity. Furthermore, they seem to be feasible model systems for metal phosphonates as they exhibit similar structural characteristics. Most of these salt-type solids show extended hydrogen-bonded networks which are characterized by a rich diversity of strong charge-supported hydrogen bonds (Aakeröy \& Seddon, 1993; Białek et al., 2013) besides some weaker intermolecular interactions (van Megen et al., 2016a,b).

A search in the Cambridge Structure Database (Groom et al., 2016) yielded more than 180 entries for the hexane-1,6diaminium dication $(H 16 A H)$. At this point it is not our aim to review all these structures, but we think it is worth highlighting some important classes of compounds and applications. The structures and properties of many simple salts of $H 16 A H$, like halides (van Blerk \& Kruger, 2008), acetates (Paul \& Kubicki, 2009) and salts with more complex inorganic anions such as hexafluoridosilcate (Ouasri et al., 2014), tetraiodide (Reiss \& van Megen, 2012) or dihydrogen arsenate (Wilkinson \& 
Harrison, 2007) have been extensively studied. Moreover, the $H 16 A H$ dication is well known for its use in crystal engineering of hydrogen-bonded solids which contain unstable species (Frank \& Reiss, 1997), in supramolecular chemistry (Assaf \& Nau, 2015), as a tecton for the construction of layered materials (Bujoli-Doeuff et al., 2012), or as a cationic template for novel complex systems (Holtby et al., 2007). Finally, it should be stressed out that the H16AH cation is applied in the context of nylon-based hybride materials (Boncel et al., 2014).<smiles>[NH3+]CCCCCC[NH3+]</smiles><smiles>O=P([O-])(O)CCCCCCP(=O)([O-])O</smiles>

This contribution is part of an ongoing study regarding the structural chemistry of alkane- $\alpha, \omega$-diphosphonic acids (van Megen et al., 2015) and their organic aminium salts (van Megen et al., 2016a,b).

\section{Structural commentary}

The asymmetric unit of $\left[\mathrm{H}_{3} \mathrm{~N}\left(\mathrm{CH}_{2}\right)_{6} \mathrm{NH}_{3}\right]\left[(\mathrm{HO}) \mathrm{O}_{2} \mathrm{P}\left(\mathrm{CH}_{2}\right)_{6}\right.$ $\left.\mathrm{PO}_{2}(\mathrm{OH})\right]$ consists of one half of an $\mathrm{H} 16 \mathrm{AH}$ dication and one half of a hexane-1,6-diyl bis(hydrogen phosphonate) dianion $(16 P H O S)$. Both ions are located around different inversion centres of space group type $P 2_{1} / c$ (Wyckoff sites $2 a$ and $2 d$, respectively). Bond lengths and angles in the dication as well as in the dianion are in the expected ranges (Table 1).
Table 1

Selected geometric parameters $\left(\AA{ }^{\circ}\right)$.

\begin{tabular}{lrlr}
\hline $\mathrm{P} 1-\mathrm{O} 3$ & $1.4977(13)$ & $\mathrm{P} 1-\mathrm{O} 1$ & $1.5817(14)$ \\
$\mathrm{P} 1-\mathrm{O} 2$ & $1.5112(13)$ & & \\
$\mathrm{O} 3-\mathrm{P} 1-\mathrm{O} 2$ & $114.23(8)$ & $\mathrm{O} 3-\mathrm{P} 1-\mathrm{C} 4$ & $111.37(9)$ \\
$\mathrm{O} 3-\mathrm{P} 1-\mathrm{O} 1$ & $111.27(8)$ & $\mathrm{O} 2-\mathrm{P} 1-\mathrm{C} 4$ & $109.71(8)$ \\
$\mathrm{O} 2-\mathrm{P} 1-\mathrm{O} 1$ & $105.83(8)$ & $\mathrm{O} 1-\mathrm{P} 1-\mathrm{C} 4$ & $103.79(8)$ \\
& & & \\
$\mathrm{N} 1-\mathrm{C} 1-\mathrm{C} 2-\mathrm{C} 3$ & $69.9(3)$ & $\mathrm{P} 1-\mathrm{C} 4-\mathrm{C} 5-\mathrm{C} 6$ & $-177.99(15)$ \\
$\mathrm{C} 1-\mathrm{C} 2-\mathrm{C} 3-\mathrm{C}^{\mathrm{i}}$ & $174.2(3)$ & $\mathrm{C} 4-\mathrm{C} 5-\mathrm{C} 6-\mathrm{C} 6^{\mathrm{ii}}$ & $178.7(2)$ \\
\hline
\end{tabular}

As shown in Fig. 1, the cation has a conformation best described as a double hook. In detail, atom $\mathrm{C} 1$ is turned out from the plane of the central four carbon atoms by about $6^{\circ}$ (Table 1), whereas atom N1 is turned out significantly from the plane defined by the central four carbon atoms [N1-C1$\left.\mathrm{C} 2-\mathrm{C} 3=69.9(3)^{\circ}\right]$. The individual conformation of the cationic diaminium tecton seems to be a compromise between an effort to form the most stable conformation on the one hand, and intermolecular interactions, namely hydrogen bonding and van der Waals interactions, on the other hand (Frank \& Reiss, 1996, 1997).

The conformation of the anion is that of the energetically most stable all-transoid conformation of the hexane-1,6-diyl moiety (r.m.s. of the six carbon atoms and two phosphorus atoms: $0.2643 \AA$ ), also expressed by the almost perfect antiperiplanar arrangement of each $\mathrm{CH}_{2}$ group ( $c f$. the torsion angles in Table 1). A detailed view of the hydrogen phosphonate groups shows the $\mathrm{P}-\mathrm{OH}$ distance of 1.5817 (14) $\AA$ to be greater than the two other $\mathrm{P}-\mathrm{O}$ distances [1.4977 (13) and $1.5112(13) \AA]$.

\section{Supramolecular features}

Within the crystal of the title compound, the aminium groups of the cations as well as the hydrogen phosphonate groups of

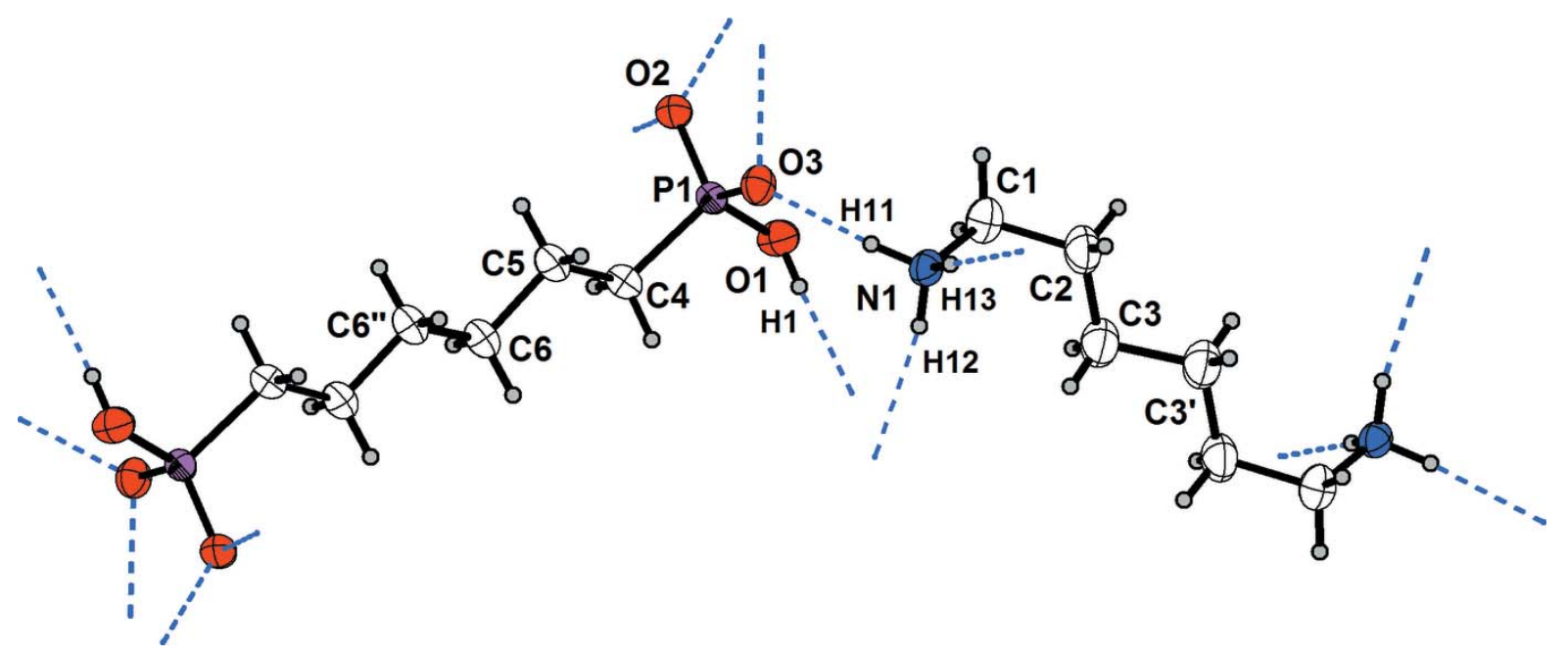

Figure 1

The $\mathrm{H} 16 \mathrm{AH}$ cation and the $16 \mathrm{PHOS}$ anion are shown together with their hydrogen bonds. Displacement ellipsoids are drawn at the $50 \%$ probability level; hydrogen atoms are drawn as spheres with arbitrary radii. [Symmetry codes: (') $-x,-y,-z ;\left(^{\prime \prime}\right) 1-x, 1-y, 1-z$.] 


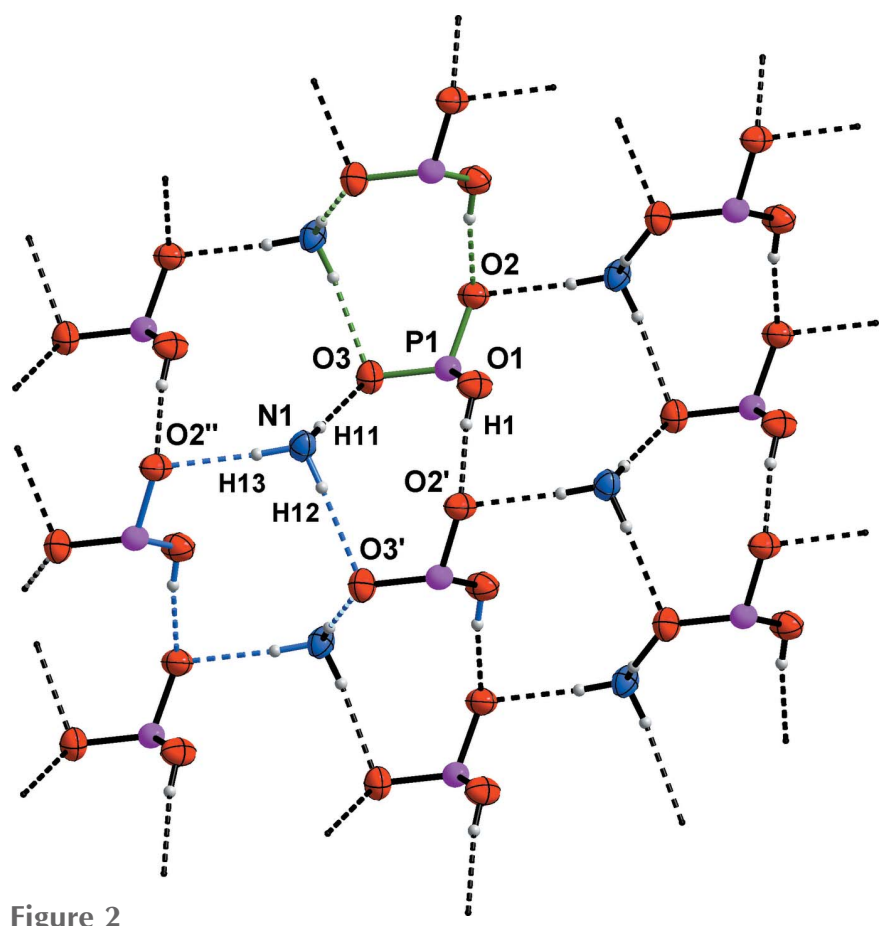

The two-dimensional hydrogen-bonded network composed of aminium and hydrogen phosphonate groups parallel to the ac plane. The $R_{3}^{3}(10)$ graph-set motif is indicated by green bonds and the $R_{5}^{4}(12)$ motif with blue bonds. [Symmetry codes: (') $x,-y+\frac{1}{2}, z-\frac{1}{2}$; (") $x-1,-y+\frac{1}{2}, z-\frac{1}{2}$.]

the anions form hydrogen bonds with adjacent ions. In detail, each hydrogen atom of the $\mathrm{NH}_{3}$ group and the $\mathrm{OH}$ group of the hydrogen phosphonate moiety donates a single hydrogen bond to a phosphoryl oxygen atom (Fig. 1), whereby each phosphoryl oxygen atom accepts two hydrogen bonds.

Anions and cations are connected by medium strong to strong, charge-supported $\mathrm{N}-\mathrm{H} \cdots \mathrm{O}$ and $\mathrm{O}-\mathrm{H} \cdots \mathrm{O}$ hydrogen bonds (Steiner, 2002; Table 2). The hydrogen-bonding interactions help to construct a two-dimensional network which propagates parallel to the $a c$ plane (Fig. 2). This network contains two characteristic types of meshes (Fig. 2), which can be classified as ten-membered and twelve-membered hydrogen-bonded ring motifs with the first level graph-set descriptors $R_{3}^{3}(10)$ and $R_{5}^{4}(12)$, respectively (Etter et al., 1990). It is remarkable that the structure of $\mathrm{NH}_{4} \mathrm{C}_{10} \mathrm{H}_{21} \mathrm{PO}_{2} \mathrm{OH}$ (Boczula et al., 2012) possesses layers with a very similar topology $\left[R_{3}^{3}(10)\right.$ and $\left.R_{5}^{5}(12)\right]$.

Along the $b$ axis of the unit cell, these hydrogen-bonded networks are linked by the alkylene chains of the anions as well as the cations, forming a three-dimensional network with a typical herringbone pattern.

We have already shown that $\alpha, \omega$-diaminiumalkane tectons support the formation of salts with tailored, linear polyiodides (Reiss \& Engel, 2002) showing a herringbone pattern with alternating cations and anions. Thus, the title structure is a further example for both the robustness of the herringbone motif and the structure-directing properties of $\alpha, \omega$-functionalized alkylene tectons.

A comparison with the 'parent' structures, namely those of 1,6-diaminohexane (Thalladi et al., 2000) and hexane-1,6-di-
Table 2

Hydrogen-bond geometry $\left(\AA{ }^{\circ}\right)$.

\begin{tabular}{lllll}
\hline$D-\mathrm{H} \cdots A$ & $D-\mathrm{H}$ & $\mathrm{H} \cdots A$ & $D \cdots A$ & $D-\mathrm{H} \cdots A$ \\
\hline $\mathrm{N} 1-\mathrm{H} 11 \cdots \mathrm{O} 3$ & $0.89(2)$ & $1.90(2)$ & $2.782(2)$ & $168(2)$ \\
$\mathrm{N} 1-\mathrm{H} 12 \cdots 3^{\prime}$ & $0.87(3)$ & $2.05(3)$ & $2.905(2)$ & $165(2)$ \\
$\mathrm{N} 1-\mathrm{H} 13 \cdots \mathrm{O} 2^{\prime \prime}$ & $0.90(2)$ & $1.94(2)$ & $2.828(2)$ & $170(2)$ \\
$\mathrm{O} 1-\mathrm{H} 1 \cdots \mathrm{O} 2^{\prime}$ & $0.81(3)$ & $1.76(3)$ & $2.5546(19)$ & $168(3)$ \\
\hline
\end{tabular}

Symmetry codes: (') $x,-y+\frac{1}{2}, z-\frac{1}{2} ;\left(^{\prime \prime}\right) x-1,-y+\frac{1}{2}, z-\frac{1}{2}$.

phosphonic acid (van Megen et al., 2015) seems useful. A characteristic feature of each herringbone motif is the angle of the fishbones to each other. It is not surprising, then, that this angle in the title crystal structure is almost the average of those found for the parent structures (Fig. 3), which is another proof of the usefulness of $\alpha, \omega$-diaminiumalkane tectons in crystal engineering.

\section{Related structures}

For related hydrogen phosphonates, phosphonates and bis(phosphonates), see: Boczula et al. (2012); Ferguson et al.
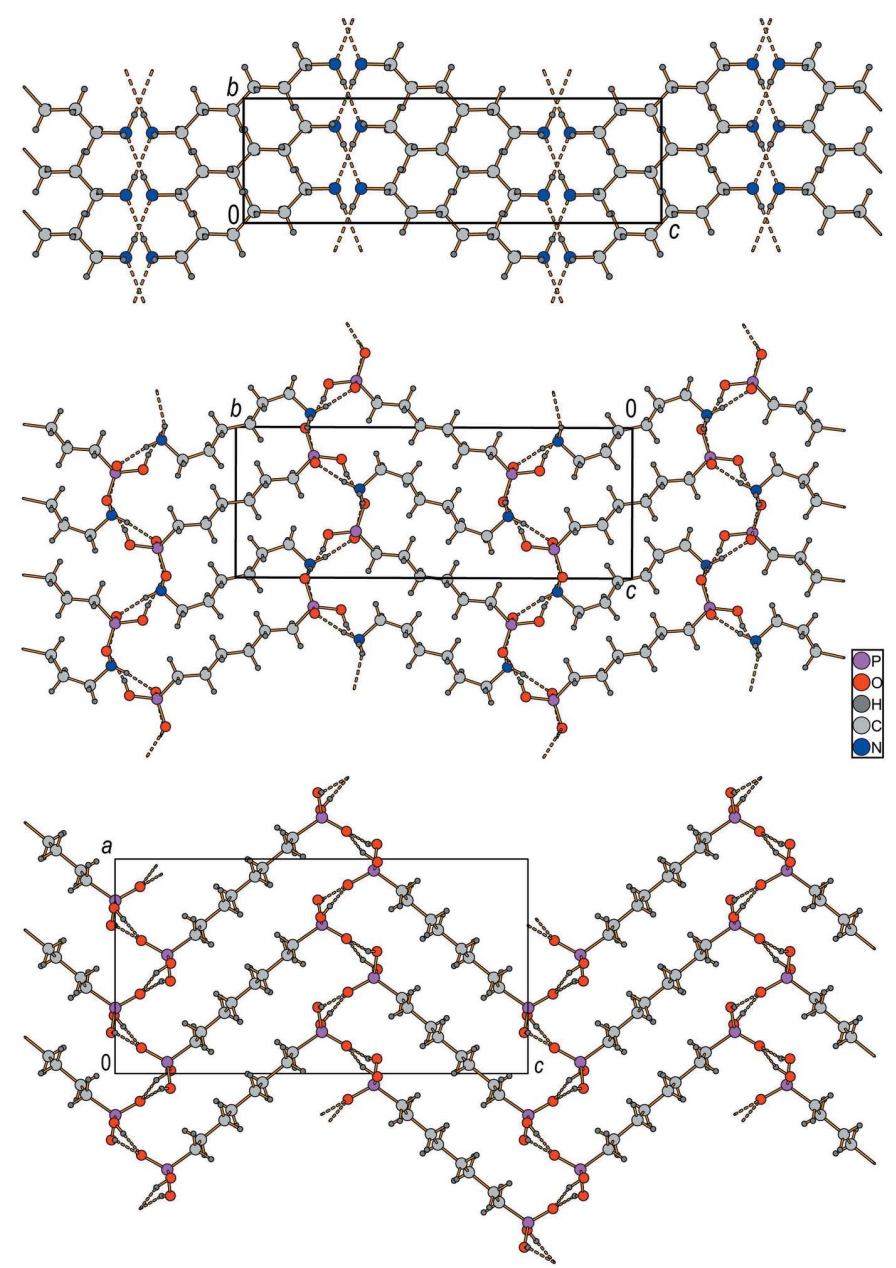

Figure 3

Comparison of the herringbone pattern of 1,6-diaminohexane (upper part), 1,6-hexane-diphosphonic acid (lower part), and the title compound (middle part). 
(1998); Fu et al. (2004); Fuller \& Heimer (1995); Glidewell et al. (2000); Kan \& Ma (2011); Mahmoudkhani \& Langer (2002a,b,c); Plabst et al. (2009); van Megen et al. (2016a,b); Wheatley et al. (2001).

For related hexane-1,6-diaminium salts, see: Assaf \& Nau (2015); Boncel et al. (2014); Bujoli-Doeuff et al. (2012); Blerk \& Kruger (2008); Frank \& Reiss (1997); Holtby et al. (2007); Wilkinson \& Harrison (2007); van Megen et al. (2015).

For closely related hydrogen-bonded compounds with a herringbone pattern, see: Thalladi et al. (2000); van Megen et al. $(2016 a)$.

\section{Synthesis and crystallization}

For the preparation of the title compound, equimolar quantities $(0.5 \mathrm{mmol})$ of hexane-1,6-diamine $(58.1 \mathrm{mg})$ and hexane1,6-bisphosphonic acid (123.1 mg) were dissolved in methanol, separately. The solutions were mixed and the resulting white precipitate was then dissolved in distilled water. Within several days, colourless crystals were obtained in an open petri dish by slow evaporation of the solvent. Hexane-1,6-diamine was purchased from commercial sources and hexane-1,6-bisphosphonic acid was synthesized according to the literature (Schwarzenbach \& Zurc, 1950; Moedritzer \& Irani, 1961; Griffith et al., 1998).

Elemental analysis: $\mathrm{C}_{12} \mathrm{H}_{32} \mathrm{~N}_{2} \mathrm{O}_{6} \mathrm{P}_{2}$ (362.33): calculated $\mathrm{C}$ 39.8, H 8.9, N 7.7; found C 39.8, H 9.7, N 8.4., m.p.: $501 \mathrm{~K}$.

\section{IR and Raman spectra}

The IR and Raman spectra of the title compound are shown in Fig. 4. The vibration spectra of the title compound are in excellent accord with those of $\mathrm{NH}_{4} \mathrm{C}_{10} \mathrm{H}_{21} \mathrm{PO}_{2} \mathrm{OH}$ (Boczula et $a l ., 2012)$. This is not particularly surprising as both structures are closely related, including the hydrogen-bonding schemes. Since Boczula et al. presented a detailed discussion of the spectra, we do not include a repeated discussion. An additional, often neglected feature of such IR spectra are the broad bands associated with the $\mathrm{O}-\mathrm{H}$ stretching vibration

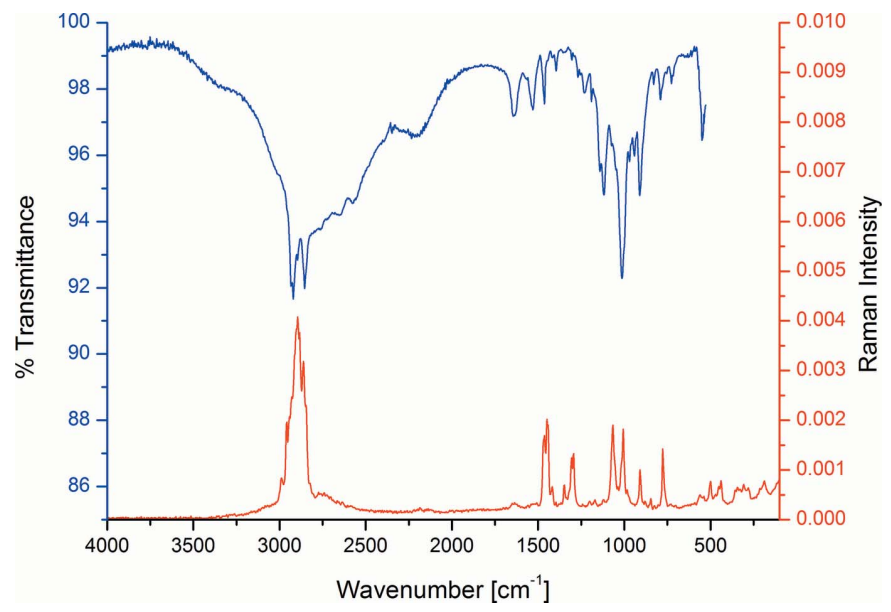

Figure 4

The IR (blue) and Raman (red) spectra of the title compound.
Table 3

Experimental details.

\begin{tabular}{|c|c|}
\hline \multicolumn{2}{|l|}{ Crystal data } \\
\hline Chemical formula & $\mathrm{C}_{6} \mathrm{H}_{18} \mathrm{~N}_{2}^{2+} \cdot \mathrm{C}_{6} \mathrm{H}_{14} \mathrm{O}_{6} \mathrm{P}_{2}^{2-}$ \\
\hline$M_{\mathrm{r}}$ & 362.33 \\
\hline Crystal system, space group & Monoclinic, $P 2_{1} / c$ \\
\hline Temperature $(\mathrm{K})$ & 292 \\
\hline$a, b, c(\AA)$ & $\begin{array}{l}5.88242(16), 20.2162(5), \\
\quad 7.7574(2)\end{array}$ \\
\hline$\beta\left(^{\circ}\right)$ & $98.090(3)$ \\
\hline$V\left(\AA^{3}\right)$ & $913.33(4)$ \\
\hline$Z$ & 2 \\
\hline Radiation type & Мо $K \alpha$ \\
\hline$\mu\left(\mathrm{mm}^{-1}\right)$ & 0.27 \\
\hline Crystal size $(\mathrm{mm})$ & $0.40 \times 0.20 \times 0.12$ \\
\hline \multicolumn{2}{|l|}{ Data collection } \\
\hline Diffractometer & $\begin{array}{l}\text { Oxford Diffraction Xcalibur with } \\
\text { Eos detector }\end{array}$ \\
\hline Absorption correction & $\begin{array}{l}\text { Multi-scan (CrysAlis PRO; Oxford } \\
\text { Diffraction, 2006) }\end{array}$ \\
\hline$T_{\min }, T_{\max }$ & $0.898,1.000$ \\
\hline $\begin{array}{l}\text { No. of measured, independent and } \\
\text { observed }[I>2 \sigma(I)] \text { reflections }\end{array}$ & 14194, 2779, 2339 \\
\hline$R_{\text {int }}$ & 0.022 \\
\hline$(\sin \theta / \lambda)_{\max }\left(\AA^{-1}\right)$ & 0.714 \\
\hline \multicolumn{2}{|l|}{ Refinement } \\
\hline$R\left[F^{2}>2 \sigma\left(F^{2}\right)\right], w R\left(F^{2}\right), S$ & $0.047,0.098,1.02$ \\
\hline No. of reflections & 2779 \\
\hline No. of parameters & 116 \\
\hline $\mathrm{H}$-atom treatment & $\begin{array}{l}\mathrm{H} \text { atoms treated by a mixture of } \\
\text { independent and constrained } \\
\text { refinement }\end{array}$ \\
\hline$\Delta \rho_{\max }, \Delta \rho_{\min }\left(\mathrm{e} \AA^{-3}\right)$ & $0.64,-0.28$ \\
\hline
\end{tabular}

Computer programs: CrysAlis PRO (Oxford Diffraction, 2006), SHELXT (Sheldrick, 2015a), SHELXL2014 (Sheldrick, 2015b), DIAMOND (Brandenburg, 2015) and publCIF (Westrip, 2010).

indicating strong hydrogen bonds (Hadži, 1965; Baran et al., 1989). A detailed discussion has also been reported very recently (van Megen et al., 2016a) for this feature. In the IR spectrum of the title compound, the maxima of the so called A, B and C bands can be estimated to be at 2750, 2200 and $1600 \mathrm{~cm}^{-1}$.

\section{Refinement}

Crystal data, data collection and structure refinement details are summarized in Table 3. All hydrogen atoms bound to either nitrogen or oxygen atoms were identified in difference syntheses and refined without any geometric constraints or restraints with individual $U_{\text {iso }}(\mathrm{H})$ values. Carbon-bound hydrogen atoms were included using a riding model (AFIX 23 option of the SHELX program for the methylene groups and AFIX 43 option for the methine groups).

\section{Acknowledgements}

We thank E. Hammes and P. Roloff for technical support. Support by the Ministry of Innovation, Science and Research of North-Rhine Westphalia and the German Research Foundation (DFG) is gratefully acknowledged (Xcalibur diffractometer; INST 208/533-1). 


\section{References}

Aakeröy, C. B. \& Seddon, K. R. (1993). Chem. Soc. Rev. 22, 397-407. Assaf, K. I. \& Nau, W. M. (2015). Chem. Soc. Rev. 44, 394-418.

Baran, J., Lis, T. \& Ratajczak, H. (1989). J. Mol. Struct. 195, 159174.

Białek, M. J., Zaręba, J. K., Janczak, J. \& Zoń, J. (2013). Cryst. Growth Des. 13, 4039-4050.

Blerk, C. van \& Kruger, G. J. (2008). Acta Cryst. C64, o537-o542.

Boczula, D., Cały, A., Dobrzyńska, D., Janczak, J. \& Zoń, J. (2012). J. Mol. Struct. 1007, 220-226.

Boncel, S., Górka, J., Shaffer, M. S. P. \& Koziol, K. K. K. (2014). Polym. Compos. 35, 523-529.

Brandenburg, K. (2015). DIAMOND. Crystal Impact GbR, Bonn, Germany.

Bujoli-Doeuff, M., Dessapt, R., Deniard, P. \& Jobic, S. (2012). Inorg. Chem. 51, 142-149.

Etter, M. C., MacDonald, J. C. \& Bernstein, J. (1990). Acta Cryst. B46, 256-262.

Ferguson, G., Glidewell, C., Gregson, R. M. \& Meehan, P. R. (1998). Acta Cryst. B54, 129-138.

Frank, W. \& Reiss, G. J. (1996). Chem. Ber. 129, 1355-1359.

Frank, W. \& Reiss, G. J. (1997). Inorg. Chem. 36, 4593-4595.

Fu, R.-B., Wu, X.-T., Hu, S.-M., Du, W.-X. \& Zhang, J.-J. (2004). Chin. J. Struct. Chem. 23, 855-861.

Fuller, J. \& Heimer, N. E. (1995). J. Chem. Crystallogr. 25, 129-136.

Glidewell, C., Ferguson, G. \& Lough, A. J. (2000). Acta Cryst. C56, $855-858$.

Griffith, J. A., McCauley, D. J., Barrans, R. E. \& Herlinger, A. W. (1998). Synth. Commun. 28, 4317-4323.

Groom, C. R., Bruno, I. J., Lightfoot, M. P. \& Ward, S. C. (2016). Acta Cryst. B72, 171-179.

Hadži, D. (1965). Pure Appl. Chem. 11, 435-445.

Holtby, A. S., Harrison, W. T. A., Yilmaz, V. T. \& Büyükgüngör, O. (2007). Solid State Sci. 9, 149-154.
Kan, W.-Q. \& Ma, J.-F. (2011). Z. Kristallogr. New Cryst. Struct. 226, 73-74.

Mahmoudkhani, A. H. \& Langer, V. (2002a). Cryst. Growth Des. 2 , 21-25.

Mahmoudkhani, A. H. \& Langer, V. (2002b). J. Mol. Struct. 609, 97108.

Mahmoudkhani, A. H. \& Langer, V. (2002c). Phosphorus Sulfur Silicon Relat. Elem. 177, 2941-2951.

Megen, M. van, Frank, W. \& Reiss, G. J. (2015). Z. Kristallogr. 230, 485-494.

Megen, M. van, Frank, W. \& Reiss, G. J. (2016a). CrystEngComm, 18, 3574-3584.

Megen, M. van, Reiss, G. J. \& Frank, W. (2016b). Acta Cryst. E72, 1456-1459.

Moedritzer, K. \& Irani, R. (1961). J. Inorg. Nucl. Chem. 22, 297-304.

Ouasri, A., Rhandour, A., Saadi, M. \& El Ammari, L. (2014). Acta Cryst. E70, o92-093.

Oxford Diffraction (2006). CrysAlis PRO. Oxford Diffraction Ltd, Abingdon, England.

Paul, A. \& Kubicki, M. (2009). J. Mol. Struct. 938, 238-244.

Plabst, M., Stock, N. \& Bein, T. (2009). Cryst. Growth Des. 9, 50495060.

Reiss, G. J. \& Engel, J. S. (2002). CrystEngComm, 4, 155-161.

Reiss, G. J. \& van Megen, M. (2012). Z. Naturforsch. Teil B, 67, 447451.

Schwarzenbach, G. \& Zurc, J. (1950). Monatsh. Chem. 81, 202-212.

Sheldrick, G. M. (2015a). Acta Cryst. A71, 3-8.

Sheldrick, G. M. (2015b). Acta Cryst. C71, 3-8.

Steiner, T. (2002). Angew. Chem. Int. Ed. 41, 48-76.

Thalladi, V. R., Boese, R. \& Weiss, H.-C. (2000). Angew. Chem. Int. Ed. 39, 918-922.

Westrip, S. P. (2010). J. Appl. Cryst. 43, 920-925.

Wheatley, P. S., Lough, A. J., Ferguson, G., Burchell, C. J. \& Glidewell, C. (2001). Acta Cryst. B57, 95-102.

Wilkinson, H. S. \& Harrison, W. T. A. (2007). Acta Cryst. E63, m902m904. 


\section{supporting information}

Acta Cryst. (2017). E73, 76-80 [https://doi.org/10.1107/S2056989016019873]

Hydrogen bonds and van der Waals forces as tools for the construction of a herringbone pattern in the crystal structure of hexane-1,6-diaminium

hexane-1,6-diyl bis(hydrogen phosphonate)

\section{Guido J. Reiss, Martin van Megen and Walter Frank}

Computing details

Data collection: CrysAlis PRO (Oxford Diffraction, 2006); cell refinement: CrysAlis PRO (Oxford Diffraction, 2006); data reduction: CrysAlis PRO (Oxford Diffraction, 2006); program(s) used to solve structure: SHELXT (Sheldrick, 2015a); program(s) used to refine structure: SHELXL2014 (Sheldrick, 2015b); molecular graphics: DIAMOND (Brandenburg, 2015); software used to prepare material for publication: publCIF (Westrip, 2010).

Hexane-1,6-diaminium hexane-1,6-diyl bis(hydrogen phosphonate)

Crystal data

$\mathrm{C}_{6} \mathrm{H}_{18} \mathrm{~N}_{2}{ }^{2+} \cdot \mathrm{C}_{6} \mathrm{H}_{14} \mathrm{O}_{6} \mathrm{P}_{2}^{2-}$

$M_{r}=362.33$

Monoclinic, $P 2_{1} / c$

$a=5.88242(16) \AA$

$b=20.2162(5) \AA$

$c=7.7574(2) \AA$

$\beta=98.090(3)^{\circ}$

$V=913.33(4) \AA^{3}$

$Z=2$

$F(000)=392$

\section{Data collection}

Oxford Diffraction Xcalibur with Eos detector diffractometer

Radiation source: (Mo) X-ray Source Detector resolution: 16.2711 pixels $\mathrm{mm}^{-1}$

$\omega$ scans

Absorption correction: multi-scan

(CrysAlis PRO; Oxford Diffraction, 2006)

$T_{\min }=0.898, T_{\max }=1.000$

\section{Refinement}

Refinement on $F^{2}$

Least-squares matrix: full

$R\left[F^{2}>2 \sigma\left(F^{2}\right)\right]=0.047$

$w R\left(F^{2}\right)=0.098$

$S=1.02$

2779 reflections
$D_{\mathrm{x}}=1.318 \mathrm{Mg} \mathrm{m}^{-3}$

Melting point: $501 \mathrm{~K}$

Mo $K \alpha$ radiation, $\lambda=0.71073 \AA$

Cell parameters from 8526 reflections

$\theta=3.0-33.9^{\circ}$

$\mu=0.27 \mathrm{~mm}^{-1}$

$T=292 \mathrm{~K}$

Block, colorless

$0.40 \times 0.20 \times 0.12 \mathrm{~mm}$

14194 measured reflections

2779 independent reflections

2339 reflections with $I>2 \sigma(I)$

$R_{\text {int }}=0.022$

$\theta_{\max }=30.5^{\circ}, \theta_{\min }=3.3^{\circ}$

$h=-8 \rightarrow 8$

$k=-28 \rightarrow 28$

$l=-11 \rightarrow 10$

116 parameters

0 restraints

Hydrogen site location: mixed

$\mathrm{H}$ atoms treated by a mixture of independent and constrained refinement 
$w=1 /\left[\sigma^{2}\left(F_{\mathrm{o}}^{2}\right)+(0.018 P)^{2}+1 . P\right]$

where $P=\left(F_{\mathrm{o}}^{2}+2 F_{\mathrm{c}}{ }^{2}\right) / 3$

$(\Delta / \sigma)_{\max }<0.001$

$$
\Delta \rho_{\max }=0.64 \mathrm{e} \AA^{-3}
$$

$\Delta \rho_{\min }=-0.28$ e $\AA^{-3}$

\section{Special details}

Geometry. All esds (except the esd in the dihedral angle between two 1.s. planes) are estimated using the full covariance matrix. The cell esds are taken into account individually in the estimation of esds in distances, angles and torsion angles; correlations between esds in cell parameters are only used when they are defined by crystal symmetry. An approximate (isotropic) treatment of cell esds is used for estimating esds involving l.s. planes.

Fractional atomic coordinates and isotropic or equivalent isotropic displacement parameters $\left(\hat{A}^{2}\right)$

\begin{tabular}{lllll}
\hline & $x$ & $y$ & $z$ & $U_{\text {iso }} / U_{\text {eq }}$ \\
\hline N1 & $-0.0383(3)$ & $0.18730(8)$ & $0.0899(2)$ & $0.0330(3)$ \\
H11 & $0.005(4)$ & $0.2266(12)$ & $0.135(3)$ & $0.043(6)^{*}$ \\
H12 & $0.024(4)$ & $0.1833(11)$ & $-0.006(3)$ & $0.047(7)^{*}$ \\
H13 & $-0.190(4)$ & $0.1883(11)$ & $0.054(3)$ & $0.043(6)^{*}$ \\
C1 & $0.0201(4)$ & $0.13413(10)$ & $0.2199(3)$ & $0.0446(5)$ \\
H1A & -0.0407 & 0.1459 & 0.3257 & $0.053^{*}$ \\
H1B & 0.1858 & 0.1315 & 0.2478 & $0.053^{*}$ \\
C2 & $-0.0708(4)$ & $0.06670(10)$ & $0.1609(3)$ & $0.0501(5)$ \\
H2A & -0.0529 & 0.0373 & 0.2607 & $0.060^{*}$ \\
H2B & -0.2338 & 0.0706 & 0.1201 & $0.060^{*}$ \\
C3 & $0.0423(4)$ & $0.03569(11)$ & $0.0201(4)$ & $0.0532(6)$ \\
H3A & 0.2072 & 0.0352 & 0.0552 & $0.064^{*}$ \\
H3B & 0.0107 & 0.0622 & -0.0848 & $0.064^{*}$ \\
P1 & $0.40040(8)$ & $0.30335(2)$ & $0.30797(6)$ & $0.02754(11)$ \\
O1 & $0.5147(2)$ & $0.23351(7)$ & $0.28712(19)$ & $0.0388(3)$ \\
H1 & $0.490(4)$ & $0.2187(12)$ & $0.190(3)$ & $0.051(7)^{*}$ \\
O2 & $0.4820(2)$ & $0.32500(6)$ & $0.49279(16)$ & $0.0357(3)$ \\
O3 & $0.1446(2)$ & $0.29998(6)$ & $0.26262(18)$ & $0.0359(3)$ \\
C4 & $0.5251(3)$ & $0.35522(9)$ & $0.1593(2)$ & $0.0367(4)$ \\
H4A & 0.6898 & 0.3568 & 0.1952 & $0.044^{*}$ \\
H4B & 0.4988 & 0.3351 & 0.0447 & $0.044^{*}$ \\
C5 & $0.4345(4)$ & $0.42525(9)$ & $0.1453(3)$ & $0.0411(4)$ \\
H5A & 0.4665 & 0.4467 & 0.2580 & $0.049^{*}$ \\
H5B & 0.2693 & 0.4243 & 0.1120 & $0.049^{*}$ \\
C6 & $0.5441(4)$ & $0.46516(10)$ & $0.0111(3)$ & $0.0457(5)$ \\
H6A & 0.7089 & 0.4664 & 0.0462 & $0.055^{*}$ \\
H6B & 0.5155 & 0.4427 & -0.1004 & $0.055^{*}$ \\
& & & &
\end{tabular}

Atomic displacement parameters $\left(\AA^{2}\right)$

\begin{tabular}{lllllll}
\hline & $U^{11}$ & $U^{22}$ & $U^{33}$ & $U^{12}$ & $U^{13}$ & $U^{23}$ \\
\hline $\mathrm{N} 1$ & $0.0291(7)$ & $0.0326(8)$ & $0.0383(9)$ & $-0.0038(6)$ & $0.0077(6)$ & $-0.0065(6)$ \\
$\mathrm{C} 1$ & $0.0472(11)$ & $0.0334(10)$ & $0.0519(12)$ & $-0.0008(8)$ & $0.0029(9)$ & $-0.0008(9)$ \\
$\mathrm{C} 2$ & $0.0533(12)$ & $0.0333(10)$ & $0.0656(15)$ & $-0.0034(9)$ & $0.0151(11)$ & $0.0004(10)$ \\
$\mathrm{C} 3$ & $0.0521(13)$ & $0.0383(11)$ & $0.0710(16)$ & $-0.0045(10)$ & $0.0152(12)$ & $-0.0072(11)$ \\
P1 & $0.0292(2)$ & $0.0280(2)$ & $0.0258(2)$ & $-0.00367(16)$ & $0.00522(15)$ & $0.00149(16)$
\end{tabular}




\begin{tabular}{lllllll} 
O1 & $0.0460(8)$ & $0.0380(7)$ & $0.0322(7)$ & $0.0093(6)$ & $0.0050(6)$ & $0.0007(6)$ \\
O2 & $0.0410(7)$ & $0.0384(7)$ & $0.0277(6)$ & $-0.0069(5)$ & $0.0052(5)$ & $-0.0010(5)$ \\
O3 & $0.0297(6)$ & $0.0351(7)$ & $0.0425(7)$ & $-0.0028(5)$ & $0.0042(5)$ & $-0.0021(6)$ \\
C4 & $0.0428(10)$ & $0.0372(9)$ & $0.0321(9)$ & $-0.0055(8)$ & $0.0119(8)$ & $0.0024(7)$ \\
C5 & $0.0538(12)$ & $0.0339(9)$ & $0.0381(10)$ & $-0.0053(8)$ & $0.0151(9)$ & $0.0054(8)$ \\
C6 & $0.0619(13)$ & $0.0365(10)$ & $0.0412(11)$ & $-0.0099(9)$ & $0.0165(10)$ & $0.0066(8)$ \\
\hline
\end{tabular}

Geometric parameters $\left(\AA,{ }^{\circ}\right)$

\begin{tabular}{|c|c|c|c|}
\hline $\mathrm{N} 1-\mathrm{C} 1$ & $1.481(3)$ & $\mathrm{P} 1-\mathrm{O} 2$ & $1.5112(13)$ \\
\hline N1-H11 & $0.89(2)$ & $\mathrm{P} 1-\mathrm{O} 1$ & $1.5817(14)$ \\
\hline $\mathrm{N} 1-\mathrm{H} 12$ & $0.87(3)$ & $\mathrm{P} 1-\mathrm{C} 4$ & 1.7907 (18) \\
\hline $\mathrm{N} 1-\mathrm{H} 13$ & $0.90(2)$ & $\mathrm{O} 1-\mathrm{H} 1$ & $0.81(3)$ \\
\hline $\mathrm{C} 1-\mathrm{C} 2$ & $1.511(3)$ & $\mathrm{C} 4-\mathrm{C} 5$ & $1.511(3)$ \\
\hline $\mathrm{C} 1-\mathrm{H} 1 \mathrm{~A}$ & 0.9700 & $\mathrm{C} 4-\mathrm{H} 4 \mathrm{~A}$ & 0.9700 \\
\hline $\mathrm{C} 1-\mathrm{H} 1 \mathrm{~B}$ & 0.9700 & $\mathrm{C} 4-\mathrm{H} 4 \mathrm{~B}$ & 0.9700 \\
\hline $\mathrm{C} 2-\mathrm{C} 3$ & $1.494(3)$ & $\mathrm{C} 5-\mathrm{C} 6$ & $1.529(3)$ \\
\hline $\mathrm{C} 2-\mathrm{H} 2 \mathrm{~A}$ & 0.9700 & $\mathrm{C} 5-\mathrm{H} 5 \mathrm{~A}$ & 0.9700 \\
\hline $\mathrm{C} 2-\mathrm{H} 2 \mathrm{~B}$ & 0.9700 & $\mathrm{C} 5-\mathrm{H} 5 \mathrm{~B}$ & 0.9700 \\
\hline $\mathrm{C} 3-\mathrm{C}^{\mathrm{i}}$ & $1.544(4)$ & $\mathrm{C} 6-\mathrm{C}^{\mathrm{ii}}$ & $1.503(4)$ \\
\hline $\mathrm{C} 3-\mathrm{H} 3 \mathrm{~A}$ & 0.9700 & C6-H6A & 0.9700 \\
\hline $\mathrm{C} 3-\mathrm{H} 3 \mathrm{~B}$ & 0.9700 & C6-H6B & 0.9700 \\
\hline $\mathrm{P} 1-\mathrm{O} 3$ & $1.4977(13)$ & & \\
\hline $\mathrm{C} 1-\mathrm{N} 1-\mathrm{H} 11$ & $110.7(14)$ & $\mathrm{O} 3-\mathrm{P} 1-\mathrm{O} 1$ & $111.27(8)$ \\
\hline $\mathrm{C} 1-\mathrm{N} 1-\mathrm{H} 12$ & $115.2(15)$ & $\mathrm{O} 2-\mathrm{P} 1-\mathrm{O} 1$ & $105.83(8)$ \\
\hline $\mathrm{H} 11-\mathrm{N} 1-\mathrm{H} 12$ & $107(2)$ & $\mathrm{O} 3-\mathrm{P} 1-\mathrm{C} 4$ & $111.37(9)$ \\
\hline $\mathrm{C} 1-\mathrm{N} 1-\mathrm{H} 13$ & 110.5 (14) & $\mathrm{O} 2-\mathrm{P} 1-\mathrm{C} 4$ & $109.71(8)$ \\
\hline $\mathrm{H} 11-\mathrm{N} 1-\mathrm{H} 13$ & $108.3(19)$ & $\mathrm{O} 1-\mathrm{P} 1-\mathrm{C} 4$ & $103.79(8)$ \\
\hline $\mathrm{H} 12-\mathrm{N} 1-\mathrm{H} 13$ & $105(2)$ & $\mathrm{P} 1-\mathrm{O} 1-\mathrm{H} 1$ & $113.8(18)$ \\
\hline $\mathrm{N} 1-\mathrm{C} 1-\mathrm{C} 2$ & $114.23(18)$ & $\mathrm{C} 5-\mathrm{C} 4-\mathrm{P} 1$ & $115.00(13)$ \\
\hline $\mathrm{N} 1-\mathrm{C} 1-\mathrm{H} 1 \mathrm{~A}$ & 108.7 & $\mathrm{C} 5-\mathrm{C} 4-\mathrm{H} 4 \mathrm{~A}$ & 108.5 \\
\hline $\mathrm{C} 2-\mathrm{C} 1-\mathrm{H} 1 \mathrm{~A}$ & 108.7 & $\mathrm{P} 1-\mathrm{C} 4-\mathrm{H} 4 \mathrm{~A}$ & 108.5 \\
\hline $\mathrm{N} 1-\mathrm{C} 1-\mathrm{H} 1 \mathrm{~B}$ & 108.7 & $\mathrm{C} 5-\mathrm{C} 4-\mathrm{H} 4 \mathrm{~B}$ & 108.5 \\
\hline $\mathrm{C} 2-\mathrm{C} 1-\mathrm{H} 1 \mathrm{~B}$ & 108.7 & $\mathrm{P} 1-\mathrm{C} 4-\mathrm{H} 4 \mathrm{~B}$ & 108.5 \\
\hline $\mathrm{H} 1 \mathrm{~A}-\mathrm{C} 1-\mathrm{H} 1 \mathrm{~B}$ & 107.6 & $\mathrm{H} 4 \mathrm{~A}-\mathrm{C} 4-\mathrm{H} 4 \mathrm{~B}$ & 107.5 \\
\hline $\mathrm{C} 3-\mathrm{C} 2-\mathrm{C} 1$ & 115.14 (19) & $\mathrm{C} 4-\mathrm{C} 5-\mathrm{C} 6$ & $111.41(17)$ \\
\hline $\mathrm{C} 3-\mathrm{C} 2-\mathrm{H} 2 \mathrm{~A}$ & 108.5 & $\mathrm{C} 4-\mathrm{C} 5-\mathrm{H} 5 \mathrm{~A}$ & 109.3 \\
\hline $\mathrm{C} 1-\mathrm{C} 2-\mathrm{H} 2 \mathrm{~A}$ & 108.5 & $\mathrm{C} 6-\mathrm{C} 5-\mathrm{H} 5 \mathrm{~A}$ & 109.3 \\
\hline $\mathrm{C} 3-\mathrm{C} 2-\mathrm{H} 2 \mathrm{~B}$ & 108.5 & $\mathrm{C} 4-\mathrm{C} 5-\mathrm{H} 5 \mathrm{~B}$ & 109.3 \\
\hline $\mathrm{C} 1-\mathrm{C} 2-\mathrm{H} 2 \mathrm{~B}$ & 108.5 & $\mathrm{C} 6-\mathrm{C} 5-\mathrm{H} 5 \mathrm{~B}$ & 109.3 \\
\hline $\mathrm{H} 2 \mathrm{~A}-\mathrm{C} 2-\mathrm{H} 2 \mathrm{~B}$ & 107.5 & $\mathrm{H} 5 \mathrm{~A}-\mathrm{C} 5-\mathrm{H} 5 \mathrm{~B}$ & 108.0 \\
\hline $\mathrm{C} 2-\mathrm{C} 3-\mathrm{C} 3^{\mathrm{i}}$ & $112.1(2)$ & $\mathrm{C} 6{ }^{\mathrm{ii}-}-\mathrm{C} 6-\mathrm{C} 5$ & $113.6(2)$ \\
\hline $\mathrm{C} 2-\mathrm{C} 3-\mathrm{H} 3 \mathrm{~A}$ & 109.2 & $\mathrm{C} 6{ }^{\mathrm{ii}}-\mathrm{C} 6-\mathrm{H} 6 \mathrm{~A}$ & 108.8 \\
\hline $\mathrm{C} 3-\mathrm{C} 3-\mathrm{H} 3 \mathrm{~A}$ & 109.2 & $\mathrm{C} 5-\mathrm{C} 6-\mathrm{H} 6 \mathrm{~A}$ & 108.8 \\
\hline $\mathrm{C} 2-\mathrm{C} 3-\mathrm{H} 3 \mathrm{~B}$ & 109.2 & $\mathrm{C} 6{ }^{\mathrm{ii}}-\mathrm{C} 6-\mathrm{H} 6 \mathrm{~B}$ & 108.8 \\
\hline 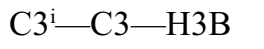 & 109.2 & $\mathrm{C} 5-\mathrm{C} 6-\mathrm{H} 6 \mathrm{~B}$ & 108.8 \\
\hline $\mathrm{H} 3 \mathrm{~A}-\mathrm{C} 3-\mathrm{H} 3 \mathrm{~B}$ & 107.9 & $\mathrm{H} 6 \mathrm{~A}-\mathrm{C} 6-\mathrm{H} 6 \mathrm{~B}$ & 107.7 \\
\hline
\end{tabular}


$\mathrm{O} 3-\mathrm{P} 1-\mathrm{O} 2$

$\mathrm{N} 1-\mathrm{C} 1-\mathrm{C} 2-\mathrm{C} 3$

$\mathrm{C} 1-\mathrm{C} 2-\mathrm{C} 3-\mathrm{C}^{\mathrm{i}}$

$\mathrm{O} 3-\mathrm{P} 1-\mathrm{C} 4-\mathrm{C} 5$

$\mathrm{O} 2-\mathrm{P} 1-\mathrm{C} 4-\mathrm{C} 5$
$114.23(8)$

$69.9(3)$

$174.2(3)$

$56.90(17)$

-70.57 (17)
$176.71(15)$

$-177.99(15)$

178.7 (2)

Symmetry codes: (i) $-x,-y,-z$; (ii) $-x+1,-y+1,-z$.

Hydrogen-bond geometry $\left(A,{ }^{\circ}\right)$

\begin{tabular}{lllll}
\hline$D-\mathrm{H} \cdots A$ & $D-\mathrm{H}$ & $\mathrm{H} \cdots A$ & $D \cdots A$ & $D-\mathrm{H} \cdots A$ \\
\hline $\mathrm{N} 1-\mathrm{H} 11 \cdots \mathrm{O} 3$ & $0.89(2)$ & $1.90(2)$ & $2.782(2)$ & $168(2)$ \\
$\mathrm{N} 1-\mathrm{H} 12 \cdots \mathrm{O} 3^{\mathrm{iii}}$ & $0.87(3)$ & $2.05(3)$ & $2.905(2)$ & $165(2)$ \\
$\mathrm{N} 1-\mathrm{H} 13 \cdots \mathrm{O} 2^{\mathrm{iv}}$ & $0.90(2)$ & $1.94(2)$ & $2.828(2)$ & $170(2)$ \\
$\mathrm{O} 1-\mathrm{H} 1 \cdots \mathrm{O} 2^{\mathrm{iii}}$ & $0.81(3)$ & $1.76(3)$ & $2.5546(19)$ & $168(3)$
\end{tabular}

Symmetry codes: (iii) $x,-y+1 / 2, z-1 / 2$; (iv) $x-1,-y+1 / 2, z-1 / 2$. 